\title{
Pathology of the Spleen in Hepatosplenic Schistosomiasis. Morphometric Evaluation and Extracellular Matrix Changes
}

\author{
Carla Rangel Leite Freitas, Aryon A Barbosa Jr, André L M Fernandes, \\ Zilton A Andrade ${ }^{+}$
}

Centro de Pesquisas Gonçalo Moniz-Fiocruz, Rua Valdemar Falcão 121, 40295-001 Salvador, BA, Brasil

Histological, ultrastructural, morphometric and immunohistochemical data obtained from the study of spleens removed by splenectomy from 34 patients with advanced hepatosplenic schistosomiasis revealed that the main alterations were congestive dilatation of the venous sinuses and diffuse thickening of the splenic cords. Splenic cord thickening was due to an increase of its matrix components, especially type IV collagen and laminin, with the conspicuous absence of interstitial collagens, either of type I or type III. Deposition of interstitial collagens (types I and III) occurred in scattered, small focal areas of the red pulp, but in the outside of the walls of the venous sinuses, in lymph follicles, marginal zone, in the vicinity of fibrous trabeculae and in sidero-sclerotic nodules. However, fibrosis was not a prominent change in schistosomal splenomegaly and thus the designation "fibro-congestive splenomegaly" seems inadequate. Lymph follicles exhibited variable degrees of atrophy, hyperplasia and fibrous replacement, sometimes all of them seen in different follicles of the same spleen and even in the same examined section. Changes in white pulp did not seem to greatly contribute to increasing spleen size and weight, when compared to the much more significant red pulp enlargement.

Key words: splenomegaly - hepatosplenic schistosomiais - extra-cellular matrix - histopathology - morphometry

Histopathology of the spleen in the so-called "congestive splenomegaly" has been little explored. Probably because the findings tend to be repetitive and non specific. One classical analysis of 86 spleens, representing chronic cases of portal hypertension due to different etiologies, showed similar morphologic changes (Moschowitz 1948). These changes have been characterized as sclerocongestive or fibro-congestive, implying that there is progressive fibrosis of the splenic cords due to the prolonged congestion and dilatation of the venous sinuses. Splenic cord thickening turns the intrasplenic circulation "closed", giving place to a rapid passage from the arterial to the venous sides of the intrasplenic circulation, thus transmitting an increased volume of blood at high pressure to the portal circulation (Andrade \& Andrade 1965). As a matter of fact, the spleen itself has been considered an arteriovenous fistula (Garnett et al. 1969).

Present material represents the part of the MSc Thesis of the first author.

${ }^{+}$Corresponding author. Fax: +55-71-356.4292. E-mail: zilton@cpqgm.fiocruz.br

Received 25 March 1999

Accepted 9 September 1999
Changes in the spleen in hepatosplenic schistosomiais exhibit a typical picture of congestive splenomegaly, similar to that associate with any type of portal hypertension of whichever etiology. Splenic cords show an increased amount of extracellular matrix, with the presence of fusiform cells, resembling ordinary connective tissue. However, the fibroblast-like cells were seen to express rich activity for acid-phosphatase and non-specific esterase, a histochemical reactivity expected for macrophages, rather than fibroblasts (Andrade \& Andrade 1965). On the other hand, although the nature of the extracellular matrix changes causing diffuse thickening of the splenic cords has been investigated with histological methods (Borojevic 1987), better characterization with more specific methods are needed.

By using scanning electron microscopy associated with morphometric techniques, Fukuda (1968) and Yamamoto (1979) observed that venous sinuses were increased in number, but occupied the same percentage area in a unit red pulp area, as in the normal spleen. Therefore, hyperplasia rather than hypertrophy, constituted the morphological basis of congestive splenomegaly. This interesting finding brought additional interest to the subject of splenic changes in congestive splenomegaly.

Present study is concerned with the nature of extracellular matrix changes and the contribution of the several splenic compartments to the spleen enlargement associated with hepatosplenic schistosomiasis. 


\section{MATERIALS AND METHODS}

Thirty-four spleens weighing from 420 to 2,540 $\mathrm{g}$ were obtained by splenectomy performed at Roberto Santos Central Hospital, Salvador, BA. The spleens were sent to the Pathology Laboratory, Gonçalo Moniz Research Center, for routine pathological examination. Splenectomy was indicated to alleviate the manifestations of portal hypertension due to hepatosplenic schistosomiasis, especially gastric hemorrhage and hypersplenism. The diagnosis of schistosomiasis was made on clinico-parasitological grounds and histologically confirmed by the finding of schistosomal pipestem fibrosis on surgical liver biopsy performed at the time of splenectomy. There were 23 males and 11 females, and their ages varied from 17 to 57 years. As controls, formalin-fixed fragments of 8 normal spleens, removed during surgery for gastric neoplasm or traumatic rupture or of the spleen, were utilized.

Histology - Several blocks from different parts of the spleens were fixed in $10 \% \mathrm{pH} 7.2$ phosphate buffered formalin, dehydrated in increasing alcohol concentrations, cleared in xylol, and embedded in paraffin. Five micrometer-thick sections were stained with hematoxylin-eosin, Sirius-red for collagen, orcein for elastic fibers, Perls' technique for iron, PAS method with and without previous diastase digestion, and the Gomori's method for reticulum.

Electron microscopy - In two controls and four splenomegaly cases, small pieces of the spleen were taken immediately after removal of the organ and fixed in iced solution of $2 \%$ gluteraldehyde in $0.1 \mathrm{~mol} /$ 1 sodium cacodylate buffer, $\mathrm{pH} 7.2$, during $1 \mathrm{hr}$. The tissue was thoroughly washed in the cacodylate buffer, and post-fixed in $2 \%$ osmium tetroxide for 1 hr. After dehydration the fragments were embedded in Poly Bed B12 resin. The blocks were submitted to ultra-thin sectioning in an automatic Reichert ultramicrotome. Selected sections were mounted in copper grids, contrasted with uranyl acetate and lead citrate solutions, and examined with a Zeiss EM-109 transmission electron microscope at $50 \mathrm{mv}$.

Immunofluorescence - Pieces from six spleens were embedded in Tissue-Tek (Milar, USA) and immediately dropped into liquid nitrogen $\left(-196^{\circ} \mathrm{C}\right)$ for $5 \mathrm{~min}$. The frozen blocks were maintained in airtight bottles at $-70^{\circ} \mathrm{C}$ until the moment of use. The blocks were cut in a cryostat at $-20^{\circ} \mathrm{C}$ and the sections submitted to immuno-fluorescence technique. The primary anti-bodies used were: rabbit anti-human g-globulin, rabbit anti-human collagens, (antitype I, type III and type IV, respectively), antifibronectin and anti-laminin. Optimal dilution for these antibodies were obtained at 1:50 and 1:100. The antibodies were kindly supplied by Dr JeanAlexis Grimaud, France, and details about their preparation and specificity appear elsewhere (Andrade \& Grimaud 1986). Commercially obtained fluoresceinated anti-rabbit IgG at 1:40 dilution was used as secondary antibody.

The same immunofluorescent technique was also applied to paraffin sections of the spleen with satisfactory results. In this case the application of primary antibodies was preceded by trypsin (Gibco $\mathrm{Brl})$ digestion $\left(0.1 \%\right.$, pH $\left.7.6,37^{\circ} \mathrm{C}\right)$, during periods of time varying from 15 to $30 \mathrm{~min}$, and the use of $0.1 \%$ Evans blue, further diluted at 1:100, to cut background fluorescence.

Morphometry - Randomly sampled 5m-thick spleen histological sections stained with either Hematoxylin and Eosin and Sirius-red for collagen were examined by semi-automatic morphometry using the Leica Q500MC Image Processing and Analysis System (Leica Cambridge, Cambridge, England). For morphometric measurements a total spleen sectional area of $1.89 \times 10^{7} \mathrm{~mm}^{2}$ per case was evaluated. The sectional area of the fibrous tissue, red stained, was directly measured and calculated as a percent of the total area examined.

The following parameters were measured: sectional areas of the white pulp, fibrous trabeculae and of the red pulp. With the values of both the pulps an index was calculated. The width of the fibrous capsule of the spleen was measured at three different regions. The $\mathrm{p}$ values smaller than 0.05 were considered statistically significant.

\section{RESULTS}

Histological examination of the spleen revealed uniform and similar findings in all cases. The red pulp exhibited dilated venous sinuses, which appeared either congested or empty, bordered by thickened and paucicellular splenic cords. Siriusred or reticulin staining sections showed a delicate net of fibers throughout the red pulp, but with irregular focal areas of fibrous thickening (Fig. 1). Distribution of these areas was at random, but there was some preference for areas at the vicinity of fibrous trabeculae and zones of focal hemorrhage, especially within and around sidero-sclerotic nodules. When the fibrous thickening became more marked, elastic fibers were also more prominent. The trabeculae did not show evident changes, their thickening being proportional to the general enlargement of the spleen. Blood vessels within the trabeculae did not show evident changes, such as sclerosis, narrowing or obstruction.

The morphology of the white pulp varied considerably. Some follicles appeared enlarged and with a wide clear center, others were atrophic and exhibited variable degrees of fibrosis (Fig. 2). Progressive fibrosis could start or be more prominent both at the periphery and the center of the follicle. Fibro- 


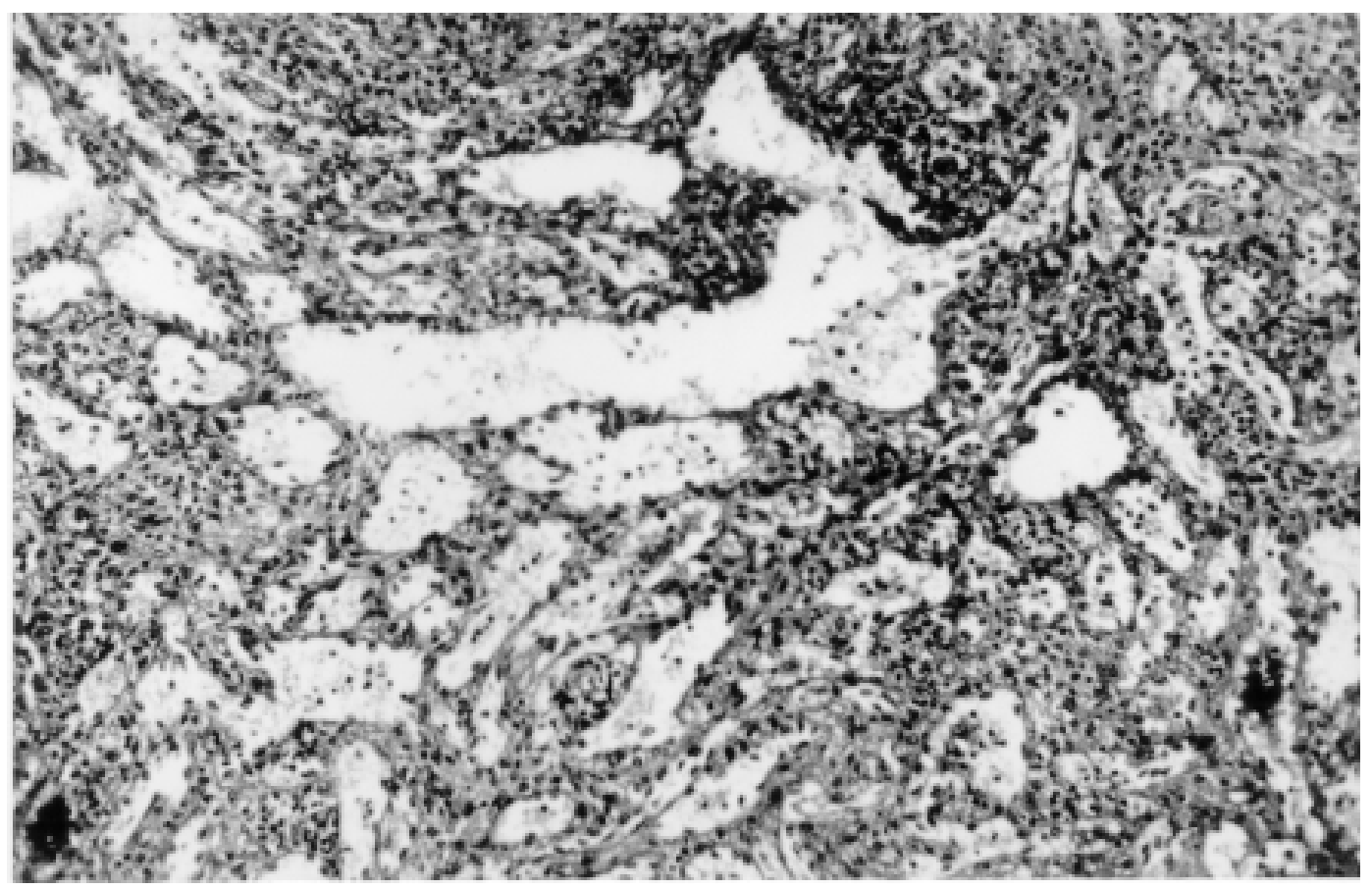

Fig. 1: spleen red pulp showing dilated venous sinuses and thickening of the splenic cords, a constant microscopic finding of schistosomal splenomegaly. $\mathrm{H} \& \mathrm{E}, 150 \mathrm{X}$.

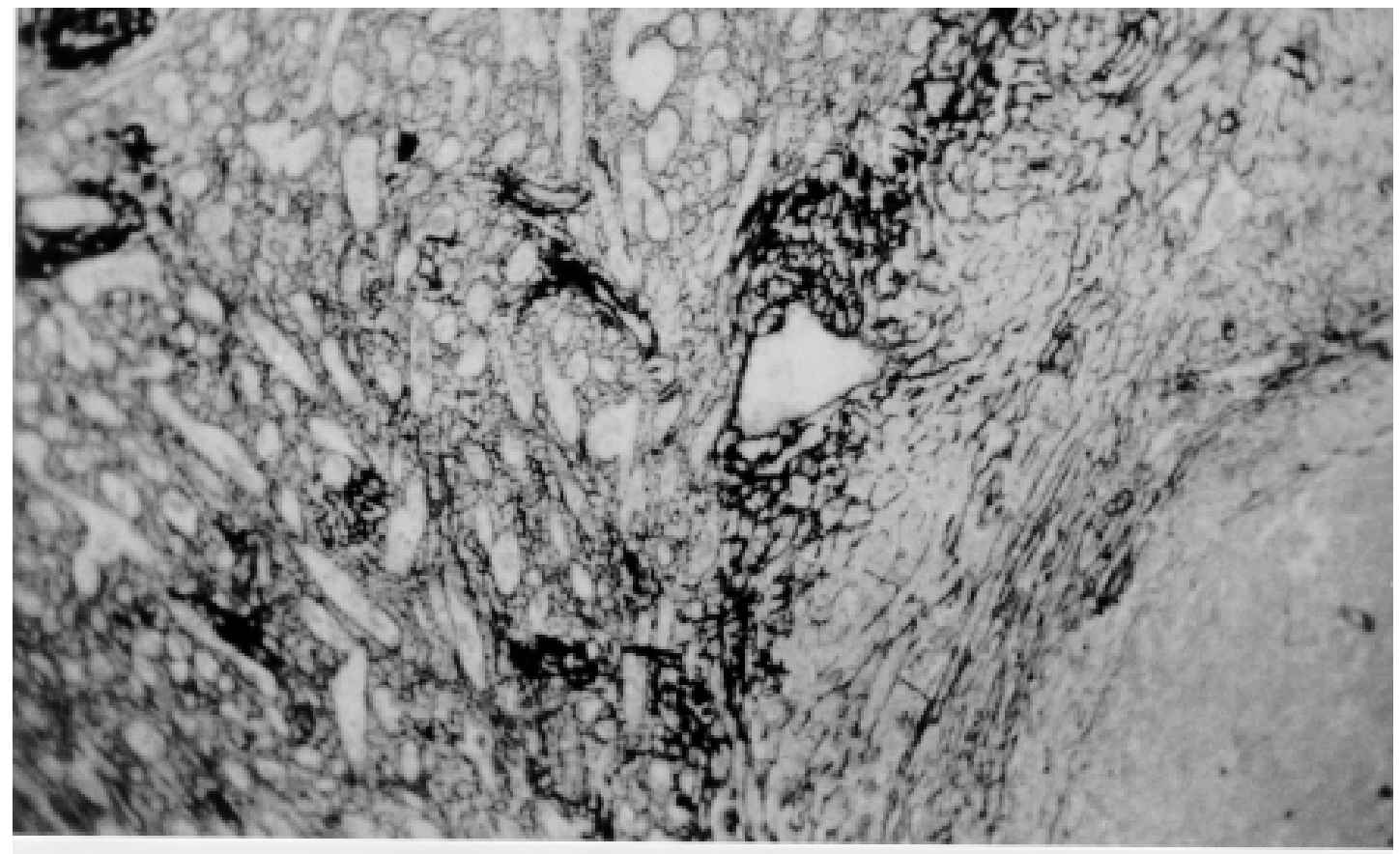

Fig. 2: dark areas of collagen deposition appear in focal distribution in the splenomegalic spleen, sparing the walls of the venous sinuses and the white pulp, which is seen at the inferior right hand corner. Sirius-red staining for collagen, $120 \mathrm{X}$. 
sis at the periphery frequently extended to the marginal zone. A deposit of hyaline material was sometimes seen around the central artery. Observation of several sites of hyaline material deposition suggested that fibrosis gradually replaced it (Fig. 3).

Immunofluorescence microscopy revealed that the trabeculae and the focal areas of thickening of the splenic cords showed positive apple-green fluorescence for type III, as well as for type I collagens, but the bulk of the splenic red pulp appeared negative for both of them. A diffuse and strong fluorescence of the red pulp was seen when the sections were treated with anti-type IV collagen (Fig. 4) and, similarly, with anti-fibronectin. The walls of the venous sinuses were clearly delineated as a continuos sinuous line by the treatment with antilaminin. The same was true for the walls of the blood vessels throughout the splenic tissue. The hyaline material seen at the center of some follicles were positive for gglobulin, which was also present within the cytoplasm of plasmocytoid cells present in many areas of the spleen. The lymph follicles exhibited a loose and delicate network of fibrils with positive immunofluorescence for type III collagen and fibronectin, while laminin and type IV collagen appeared only in the central-follicular artery wall. However, when different degrees of fibrous thick- ening involved the follicles, strong positive fluorescence was detected for type I and type III collagen, and fibronectin (Fig. 5).

Ultrastructurally, the wall of the venous sinuses appeared delimited by a continuous basement membrane that was twice thicker than normal. This membrane frequently appeared duplicated, triplicated or quadruplicated (Fig. 6). This membrane was uniform, did not present humps or fragmentation. It was limited on both sides by an amorphous or finely fibrillar material, probably proteoglycans and collagen-associated proteins, which were also present in the normal control. In some sections the venous wall appeared immersed within a densely packed collagen network, but its structure was maintained (Fig. 7), the collagen fibers accumulated in the outside. The venous sinuses were surrounded by large clear cells with a few organelles and a clear rounded nucleus (splenocytes), and, eventually, macrophages, plasmocytes and lymphocytes.

Morphometric analysis showed that the compartment occupied by the red pulp was greater in the schistosomiasis cases $\left[\right.$ mean $=1.46 \times 10^{7} \pm 7.6 \mathrm{x}$ $10^{5} \mathrm{~mm}^{2}(77.3 \pm 4 \%$ of the total sectional area $)(\mathrm{p}=$ $0.0009)]$ than in the normal control cases [mean $=$ $\left.1.33 \times 10^{7} \pm 6.1 \times 10^{5} \mathrm{~mm}^{2}(70.6 \pm 3 \%)\right]$, as was the case for the fibrous trabeculae $\left[\right.$ mean $=1.97 \times 10^{6} \pm$

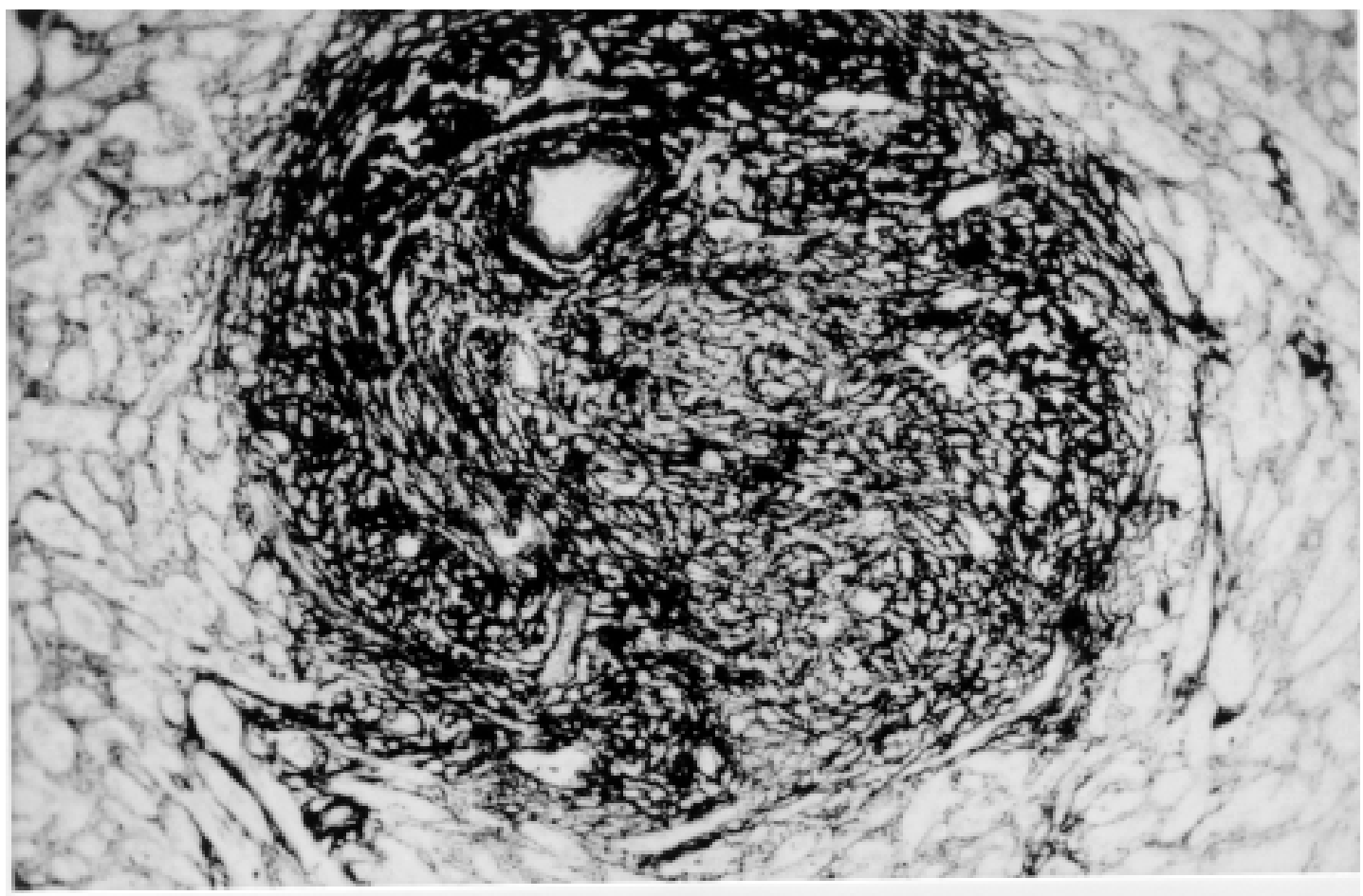

Fig. 3: total replacement of the splenic lymph follicle by dense fibrosis. The red pulp which appears around is not fibrosed. Sirius-red staining for collagen, $150 \mathrm{X}$. 


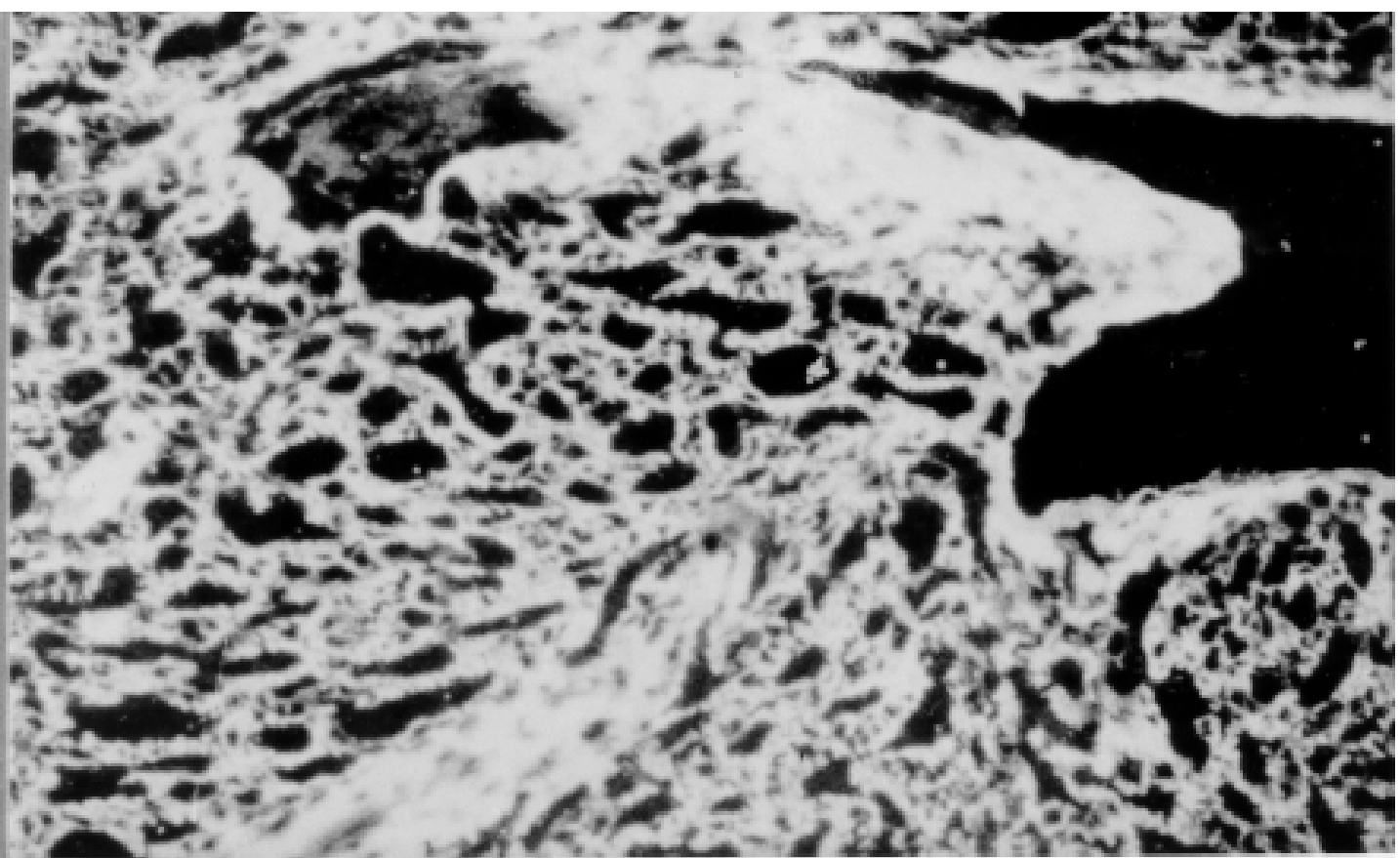

Fig. 4: the splenic cords of the red pulp appear intensely and diffusely stained for type IV collagen. Immunofluorescence microscopy, $150 \mathrm{X}$.

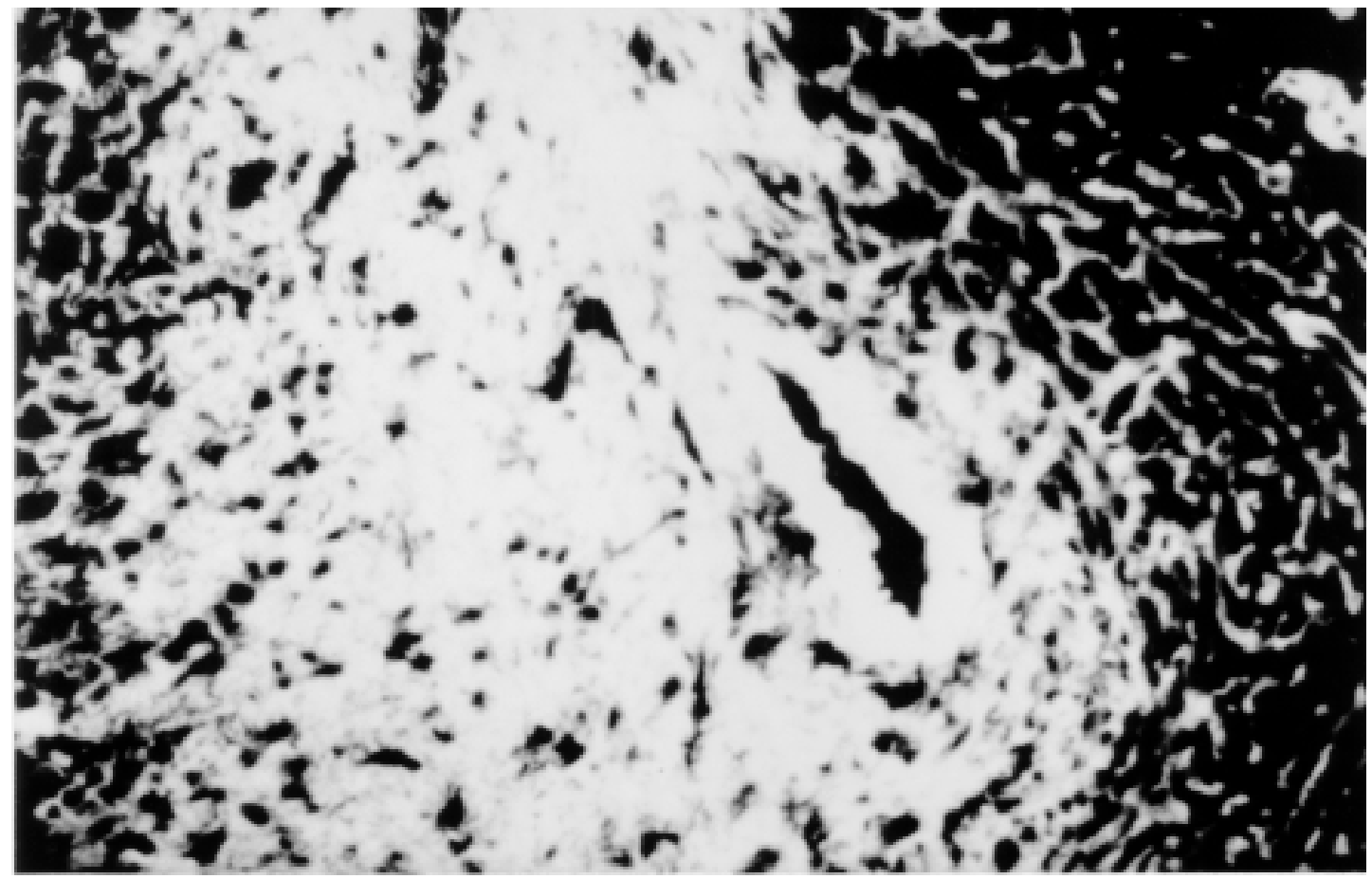

Fig. 5: type I collagen is densely deposited within a lymph follicle of the spleen, and also to some red pulp cords of the marginal zone. Immunofluorescence microscopy, $150 \mathrm{X}$. 


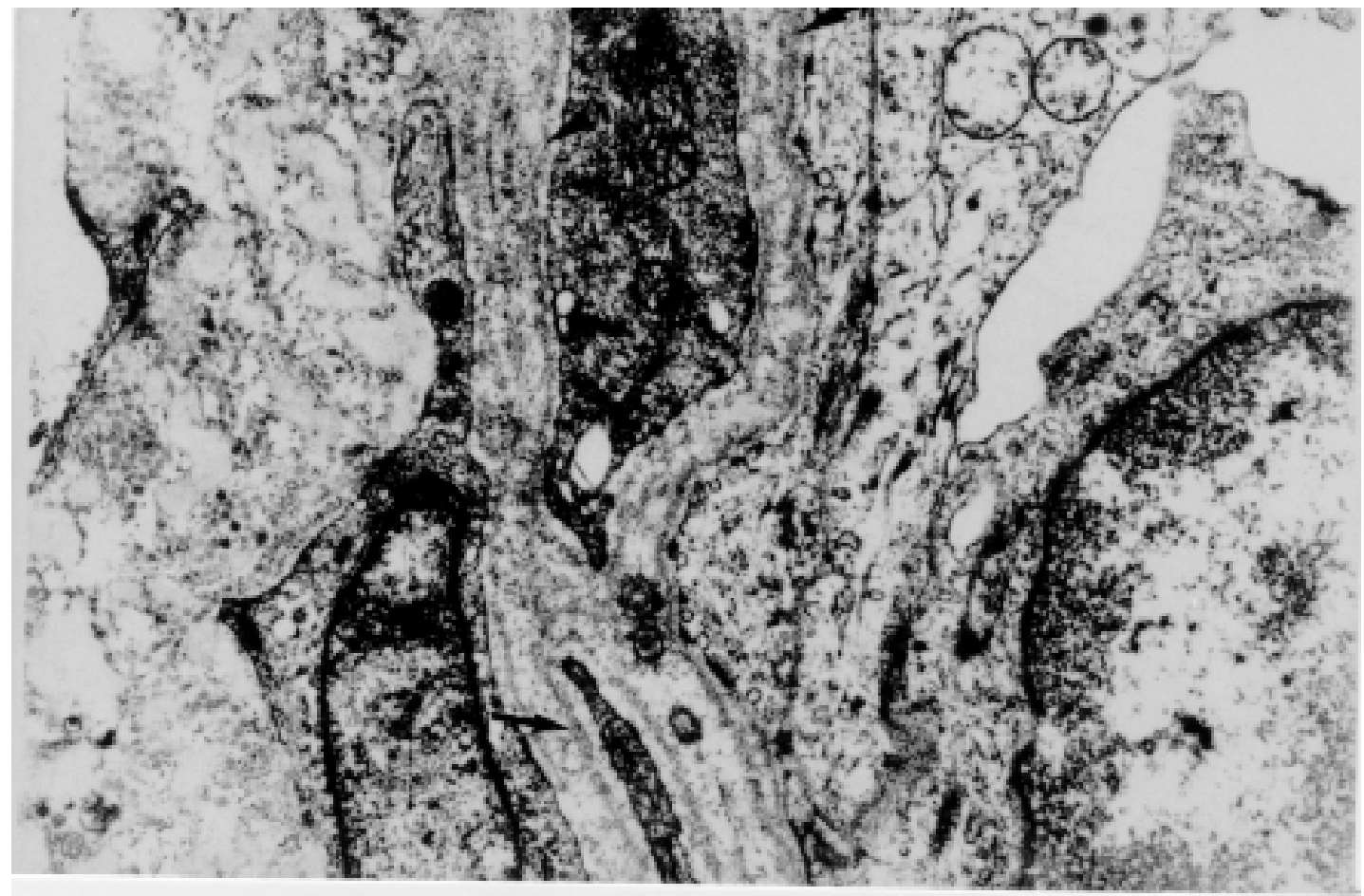

Fig. 6: wall of the venous sinus of the spleen disclosing duplication of the basement membrane-like material in its core (arrows). Electron micrograph, 7,000 X.

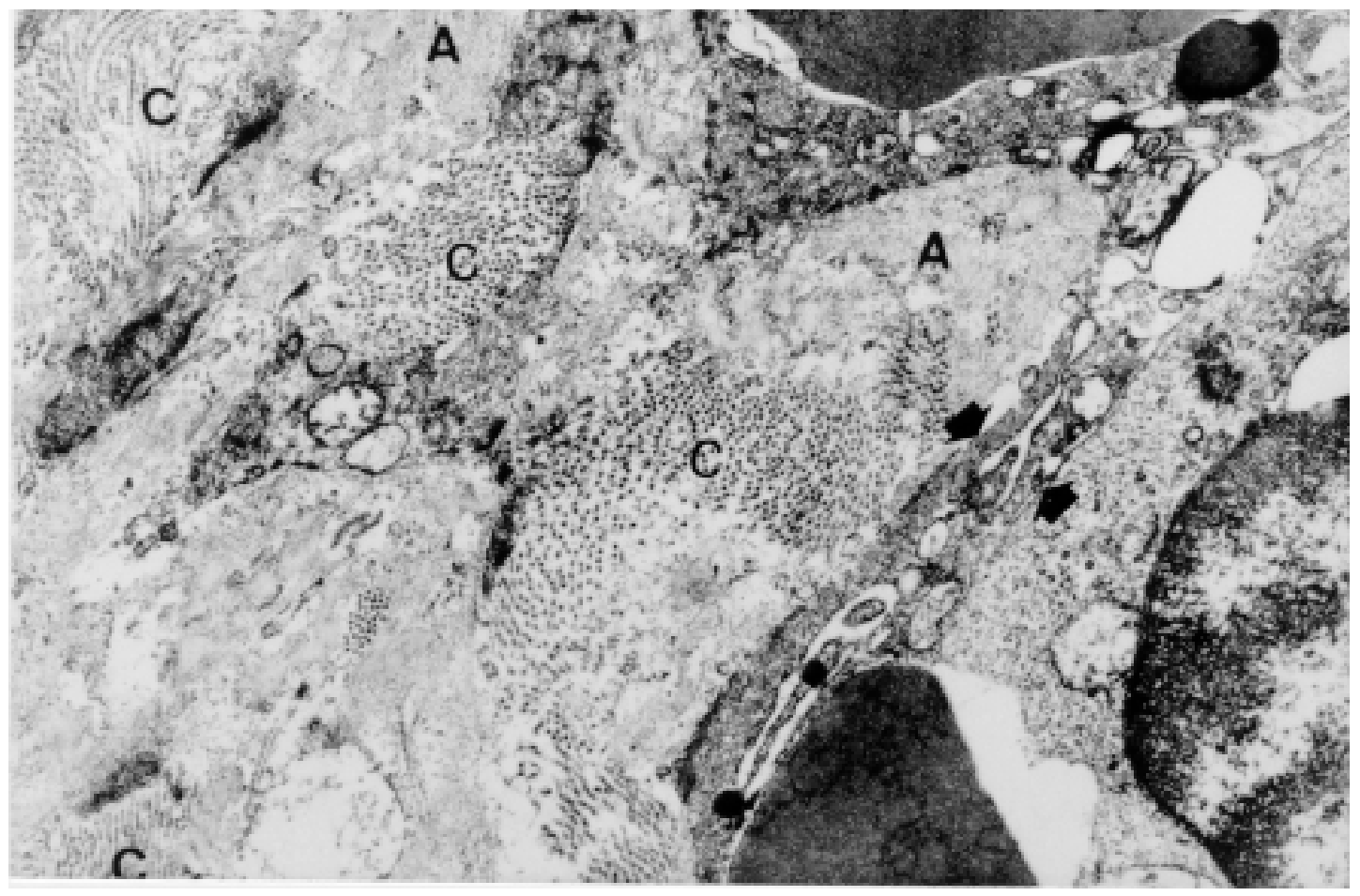

Fig. 7: focal deposition of excess extracellular matrix in the red pulp of the spleen. Collagen fibers (C) and excess amorphous material (A), probably proteoglycans appear outside of and compressing the sinus walls (arrows). Electron micrograph, 3,000 X. 
$\left.3.57 \times 10^{5} \mathrm{~mm}^{2}(10.4 \pm 2 \%)(\mathrm{p}=0.0059)\right]$ and for the index RP/WP [mean $=6.9 \pm 2.57$ ( $\mathrm{p}=0.0014)$. The compartment occupied by the white pulp, however, was smaller in the schistosomiasis cases [mean $=2.34 \mathrm{x}$ $\left.10^{6} \pm 6.89 \times 10^{5} \mathrm{~mm}^{2}(12.3 \pm 3.6 \%)(\mathrm{p}=0.0001)\right]$ than in the normal control cases [mean $=4.03 \times 10^{6} \pm 7.1 \mathrm{x}$ $\left.10^{5} \mathrm{~mm}^{2}(21.3 \pm 3.8 \%)\right]$. No statistical differences were observed for the capsule width [mean of the schistosomiasis cases $=875.4 \pm 590 \mathrm{~mm}$; mean of the normal control cases $=533.3 \pm 254 \mathrm{~mm}(\mathrm{p}=0.53)]($ Table $)$.

\section{DISCUSSION}

The histopathology of the spleen in schistosomal splenomegaly has been described by several authors (Koppisch 1941, Magalhães Filho \& Coutinho-Abath 1960, Andrade \& Andrade 1965, Fukuda 1968, Yamamoto 1979). These studies contributed not only to morphology, but also to establish the main lines of splenic physio-pathology in congestive splenomegaly. Thickening of the splenic cords and dilatation of the venous sinuses were listed as the two main microscopic findings, which indicate that the enlargement of the organ resulted from a combination of cellular proliferation and chronic passive congestion. Cellular proliferation was more evident at the early phase of the process, as shown experimentally (Andrade \& Andrade 1962). It has been determined that congestion alone is not enough to increase the spleen size beyond one and a half times (Mc Michel 1934). Thickening of the splenic cords was ascribed to fibrosis (Koppisch 1941, Borojevic 1987), which is implicit in the terms "sclero-congestive" and "fibro-congestive", with which this type of splenomegaly is designated.

Present ultrastructural and immuno-histochemical findings reveal that fibrosis, defined as deposition of excess fibrillar collagens, has only a focal, scattered distribution in schistosomal splenomegaly. The diffuse thickening of the splenic cords results from an increase of its normal components, that is, the axis represented by the basement membrane-like material and its associated glyco-proteins and proteoglycans, which form an intensely argirophilic extracellular network. The splitting and thickening of the basement membrane-like material and the increase of its associated amorphous material, is probably a response to chronic passive congestion and increased blood pressure within the venous sinuses, since the thickness and general structure of blood vessels reflect their internal pressure. These diffuse changes in the walls of splenic cords are responsible for the elastic rigidity of the spleen.

Of the several components of the spleen, only the red pulp appears as the fundamental site of changes to explain organ enlargement. The capsule, septa and trabeculae are thickened proportionally to the general increase in spleen weight and volume. This process is in keeping with the general knowledge that nature tends always to maintain the usual proportion stroma/parenchyma when an organ undergoes enlargement (Perez-Tamayo 1979).

The white pulp exhibits several changes, but these are unpredictable and variable, probably only of ancillary importance in relation to increase in volume, weight and consistency of spleen in splenomegaly. However, if one considers the overall weight of the spleen the white pulp also is increased in relation to normal.

Morphometry showed that the great spleen enlargement is mostly due to the congestive expansion of the red pulp coupled with enlargement of the fibrous trabeculae, with relative, but not absolute, diminution of the white pulp. The suggestion made by Japanese investigators (Fukuda 1968, Yamamoto 1979) that hyperplasia of the red pulp alone is responsible for splenomegaly is not supported by the present findings. Of course some degree of hyperplasia, as well as hypertrophy, cannot be ruled out. Otherwise the wall of the sinuses would be thinner than normal if only distention was

TABLE

Compartment occupied by the red pulp, white pulp and fibrous trabeculae in $1.89 \times 10^{7} \mathrm{~mm}^{2}$ of spleen section of schistosomiasis cases and normal control cases

\begin{tabular}{|c|c|c|c|c|c|}
\hline & $\begin{array}{c}\text { Schistosomiasis } \\
\mathrm{n}=13\end{array}$ & $\%$ & $\begin{array}{c}\text { Normal control } \\
\mathrm{n}=8\end{array}$ & $\%$ & \\
\hline $\begin{array}{l}\text { Red pulp } \\
\left(\mathrm{x} 10^{7} \mathrm{~mm}^{2}\right) \\
\mathrm{p}<0.01\end{array}$ & $1.460 \pm 0.760$ & $77.3 \pm 4$ & $1.336 \pm 0.606$ & $70.6 \pm 3$ & $\begin{array}{r}\mathrm{T}=3.1 \\
\text { d.f. }=19\end{array}$ \\
\hline $\begin{array}{l}\text { White pulp } \\
\left(\mathrm{x} 10^{7} \mathrm{~mm}^{2}\right) \\
\mathrm{p}<0.001\end{array}$ & $0.234 \pm 0.689$ & $12.4 \pm 3.6$ & $0.403 \pm 0.712$ & $21.3 \pm 3.8$ & $\begin{array}{r}\mathrm{T}=5.4 \\
\text { d.f. }=19\end{array}$ \\
\hline $\begin{array}{l}\text { Fibrous } \\
\text { trabeculae } \\
\left(\mathrm{x} 10^{7} \mathrm{~mm}^{2}\right)\end{array}$ & $0.196 \pm 0.036$ & $10.4 \pm 1.9$ & $0.152 \pm 0.025$ & $8 \pm 1.3$ & $\begin{array}{r}\mathrm{T}=3.1 \\
\text { d.f. }=19 \\
\mathrm{p}<0.01\end{array}$ \\
\hline
\end{tabular}


responsible for the general enlargement of the spleen. There is experimental evidence of an early phase of cellular multiplication followed by a second phase of congestion in the pathogenesis of splenomegaly of the mouse infected with Schistosoma mansoni (Andrade \& Andrade 1962). As a matter of fact, the most considerable splenomegalies results from cellular proliferation, not congestion, such as can be observed in lymphomas and visceral leishmaniasis. But prominent congestive changes probably contributes to make the participation of cellular hyperplasia histologically less evident histologically

However, present morphometric data do indicate that at least the bulk of the spleen enlargement results from an increase in the size of individual venous sinuses, by dilatation of its lumen and thickening of its walls.

\section{ACKNOWLEDGEMENTS}

To Dr Olindo Martins Filho, from the Centro de Pesquisas Renë Rachou-Fiocruz, Belo Horizonte, MG, who kindly supplied paraffin blocks from normal spleens and Dr Jean-Alexis Grimaud, École de Medicine, Université Paris VI, Paris, France, who donated the antibodies used in this study.

\section{REFERENCES}

Andrade ZA, Andrade SG 1962. Aspectos experimentais da esplenomegalia da esquistossomose. Rev Inst Med Trop São Paulo 4: 249-255.

Andrade ZA, Andrade SG 1965. Patologia do baço na esquistossomose hépato-esplênica. Rev Inst Med
Trop São Paulo 7: 218-227.

Andrade ZA, Grimaud JA 1986. Evolution of the schistosomal hepatic lesions in mice after curative chemotherapy. Am J Pathol 124: 59-65.

Borojevic R 1987. Splenic fibrosis in patients with chronic schistosomiasis. Mem Inst Oswaldo Cruz 82 (Suppl. IV): 253-255.

Fukuda Y 1968. Pathological study of Banti' syndrome. Acta Pathol Jap 18: 457-472.

Garnett ES, Goddard BA, Markby D, Webber CE 1969. The spleen as an arteriovenous shunt. The Lancet $i$ : 386-388.

Koppish E 1941. Studies on schistosomiasis mansoni in Puerto Rico. Morbid anatomy of the disease as seen in Puerto Ricans. Puerto Rico J Pub Health \& Trop. Med 16: 395-455.

Magalhães $\mathrm{F}^{\circ} \mathrm{A}$, Coutinho-Abath E 1960. Lesões esplênicas iniciais na esquistossomose mansônica humana. Rev Inst Med Trop São Paulo 2: 251259.

Mc Michael J 1934. The pathology of hepatolienal fibrosis. J Pathol Bacteriol 39: 481-502.

Moschowitz E 1948. The pathogenesis of splenomegaly in hypertension of the portal circulation, "congestive splenomegaly". Medicine 27: 187-221.

Perez-Tamayo R 1965. Some aspects of connective tissue of the liver, p. 192-210. In H Popper \& F Schaffner (eds), Progress in Liver Diseases, Churchill \& Livingstone, London.

Yamamoto K 1979. Morphological studies of the spleen in idiopathic portal hypertension (so-called Banti's syndrome without liver cirrhosis) using light microscopy, scanning electron microscopy and histometry. Acta Pathol Jap 29: 1-19. 